\title{
National survey of paediatric audiological services for diagnosis and intervention in the South African private health care sector
}

\author{
Authors: \\ Miriam E. Meyer ${ }^{1}$ \\ De Wet Swanepoel ${ }^{1,2,3}$ \\ Talita le Roux ${ }^{1}$ \\ Affiliations: \\ ${ }^{1}$ Department of Speech- \\ Language Pathology \& \\ Audiology, University of \\ Pretoria, South Africa \\ ${ }^{2}$ Ear Sciences Centre, School \\ of Surgery, University of \\ Western Australia, Australia \\ ${ }^{3}$ Ear Science Institute \\ Australia, Subiaco, Australia \\ Correspondence to: \\ De Wet Swanepoel \\ Email: \\ dewet.swanepoel@up.ac.za \\ Postal address: \\ Private Bag X20, Hatfield \\ 0028, South Africa \\ Dates: \\ Received: 26 Feb. 2014 \\ Accepted: 28 June 2014 \\ Published: 11 Nov. 2014 \\ How to cite this article: \\ Meyer, M.E., Swanepoel, \\ D., \& Le Roux, T. (2014). \\ National survey of paediatric \\ audiological services for \\ diagnosis and intervention \\ in the South African \\ private health care sector. \\ South African Journal of \\ Communication Disorders, \\ 61(1), Art. \#62, 8 pages. \\ http://dx.doi.org/10.4102/ \\ sajcd.v61i1.62

\section{Copyright:} \\ (C) 2014. The Authors. \\ Licensee: AOSIS \\ OpenJournals. This work \\ is licensed under the \\ Creative Commons \\ Attribution License.

Objective: A national survey of early hearing detection and intervention services was undertaken to describe the current status of diagnostic and intervention services in the South African private health care sector.

Methods: All private hospitals with obstetric units $(n=166)$ were surveyed telephonically. The data was integrated with data collected from self-administered questionnaires subsequently distributed nationally to private audiology practices providing hearing screening at the respective hospitals reporting hearing screening services $(n=87)$. Data was analysed descriptively to yield national percentages and frequency distributions.

Results: Average reported age at diagnosis was 11 months. Most participants (74\%) indicated that less than $20 \%$ of infants fitted with hearing aids received amplification before the age of 6 months. Most (64\%) participants indicated that the average period between confirmed diagnosis and hearing aid fitting was 1 month, on par with international benchmarks. Only $16 \%-23 \%$ of participants included all diagnostic procedures recommended by the Health Professions Council of South Africa's 2007 position statement for minimum diagnostic test batteries for infants and young children.

Conclusions: Diagnosis of hearing loss, hearing aid fitting and audiological intervention is delayed significantly in the South African private health care sector. Improved services should include integrated systematic hospital-based screening as part of birthing packages with diagnostic referral to specialist paediatric audiologists for accurate assessment and management of patients in a timely manner.

\section{Introduction}

Every day an estimated 17 babies in South Africa will be born with or acquire significant permanent bilateral hearing loss within the first few weeks of life (Swanepoel, Störbeck \& Friedland, 2009). This is based on an estimated incidence of $6 / 1000$ live births in the public health care sector, which serves $85 \%$ of the South African population, and 3/1000 live births in the private health care sector, which serves the remaining $15 \%$ of the population (Swanepoel et al., 2009).

Unlike many other congenital or early-onset disabilities, infants with hearing loss could develop speech and language skills on par with normal hearing peers, provided that the loss is identified and intervention initiated early enough (Nelson, Bougatsos \& Nygren, 2008; Watkin et al., 2007). If these infants were screened shortly after birth, audiological and medical evaluations were conducted before 3 months of age, confirmation of permanent congenital or early-onset hearing loss was no later than 4 months of age and intervention was initiated before 6 months of age, they would have had the potential to develop age-appropriate speech and language skills (Boudewyns et al., 2011; Health Professions Council of South Africa [HPCSA], 2007; JCIH, 2007; Yoshinaga-Itano \& Thomson, 2008).

However, if these children are not identified early, it could cause developmental delays in speech, language, social, emotional and cognitive development (HPCSA, 2007; Korver et al., 2010; Olusanya, 2008; Olusanya \& Newton, 2007; Yoshinaga-Itano \& Thomson, 2008). The longer the hearing loss goes undetected, the poorer speech, language, literacy and educational outcomes are likely to be for the child, with higher associated long-term costs to the family, society and the country (Joint Committee on Infant Hearing [JCIH], 2007; Korver et al., 2010; Morton \& Nance, 2006; Olusanya, 2008; Olusanya \& Newton, 2007; Olusanya, Ruben \& Parving, 2006; Swanepoel, 2008; Swanepoel, 2010; Yoshinaga-Itano \& Thomson, 2008). Ultimately, infants with hearing loss are often consigned to a life of exclusion and stigmatisation (Olusanya et al., 2006; Swanepoel, 2008; Swanepoel, 2010). 
In South Africa, infant hearing loss is primarily detected passively when parents become concerned about speech and language delays or unusual behaviour, which usually occur after 2 years of age, when the critical age of language development has passed (Swanepoel, 2008; Swanepoel et al., 2009; Swanepoel, 2010; Swanepoel, Johl \& Pienaar, 2013; Theunissen \& Swanepoel, 2008). Recent studies performed on urban samples from two provinces reported a mean age at hearing loss diagnosis of 23 and 31 months, respectively, and a mean age at initial hearing aid fitting of 28 and 39 months, respectively (Van der Spuy \& Pottas, 2008; Venter \& Viljoen, 2008). In these urban samples, it was furthermore reported that the mean age at initial enrolment into an early intervention programme was 31 months and 43 months, respectively (Van der Spuy \& Pottas, 2008; Venter \& Viljoen, 2008). In a more recent study at a diagnostic referral clinic, nearly half $(47 \%)$ of participants were only diagnosed after 36 months of age although $40 \%$ of them were suspected of having a hearing loss before 12 months of age (Swanepoel et al., 2013).

The delayed age at diagnosis could partly be attributed to the poor status of current early hearing detection services in South Africa (Meyer \& Swanepoel, 2011; Meyer, Swanepoel, Le Roux \& Van der Linde, 2012; Swanepoel et al., 2013; Theunissen \& Swanepoel, 2008). In the public sector, only $7.5 \%$ of hospitals provide some form of neonatal and infant hearing screening, whilst less than $1 \%$ offer universal screening (Theunissen \& Swanepoel, 2008). Although screening coverage might have improved since the Theunissen and Swanepoel (2008) publication, no updated survey on the current status has been published since. In the private sector, universal newborn hearing screening is only offered in 14\% of obstetric units (Meyer \& Swanepoel, 2011).

Other possible reasons for late diagnosis and enrolment into early intervention programmes in the public health care sector are poor follow-up return rates, a lack of awareness amongst parents of the importance of infant hearing screening and the detrimental effects of permanent childhood hearing impairment, and a possible delay in hearing aid procurement (Swanepoel, Ebrahim, Joseph \& Friedland, 2007; Theunissen \& Swanepoel, 2008).

Until recently, however, there has been no national survey of early hearing detection and intervention (EHDI) services in the private health care sector (Meyer et al., 2012; Meyer \& Swanepoel, 2011; Swanepoel et al., 2009). As the private health care sector serves a minority $(15 \%)$ of the South African population but comprises the majority of national health care expenditure (Dambisya, Modipa \& Health Systems Research Group, 2009), it may appear as though more resources are available for EHDI services in this sector as opposed to the public health care sector. However, screening programmes in these private health care institutions are mostly dependent on individual initiatives from audiologists in private practice who may face similar problems with programme implementation as the public health care sector (Blecher \& Harrison, 2006; Dambisya et al., 2009; Swanepoel et al., 2009). The Health Professions Council of South Africa's (HPCSA) position statement on EHDI programmes in South Africa (HPCSA, 2007) specifies recommended diagnostic protocols, practices and targets for EHDI programmes. To date it is unknown whether audiologists in private practice are aware of the HPCSA position statement and its recommended guidelines for best practice or to what extent they adhere to these guidelines. In light of this, the objective of the current study was to describe the audiological diagnostic protocols and intervention practices implemented in the South African private health care sector. The study is part of the first national survey on EHDI services in the South African private health care sector (Meyer et al., 2012; Meyer \& Swanepoel, 2011). A review of existing audiological diagnostic and intervention practices in EHDI provides baseline information essential for planning and coordination of improved widespread early hearing detection, diagnosis and intervention services into the future.

\section{Methodology Objectives}

This study had the following objectives with regard to the South African private health care sector:

- to describe diagnostic audiological protocols for EHDI

- to determine the average age at diagnosis

- to determine the average age at hearing aid fitting

- to determine the delay from diagnosis to audiological intervention

- to describe intervention practices.

\section{Research design}

A descriptive survey design was used in this national survey of the South African private health care sector.

\section{Ethical clearance}

Institutional ethical clearance was obtained from the Faculty of Humanities at the University of Pretoria before data collection was initiated. Informed consent was obtained from and confidentiality was ensured to all participating individuals and institutions.

\section{Inclusion criteria}

The total population included ward matrons at all private health care hospitals or clinics that offer obstetric services as well as the audiologists in private practice (registered with the HPCSA) who provide infant hearing screening services at these units (Figure 1). All private health care institutions that did not provide obstetric services and all audiologists in private practice working at these institutions were excluded from the study.

All private health care (non-government funded) institutions in South Africa were contacted telephonically in order to determine whether they provided obstetric services. A total of 304 such institutions, including hospitals, clinics and private practices listed in the national registry (Medpages, 2009), 


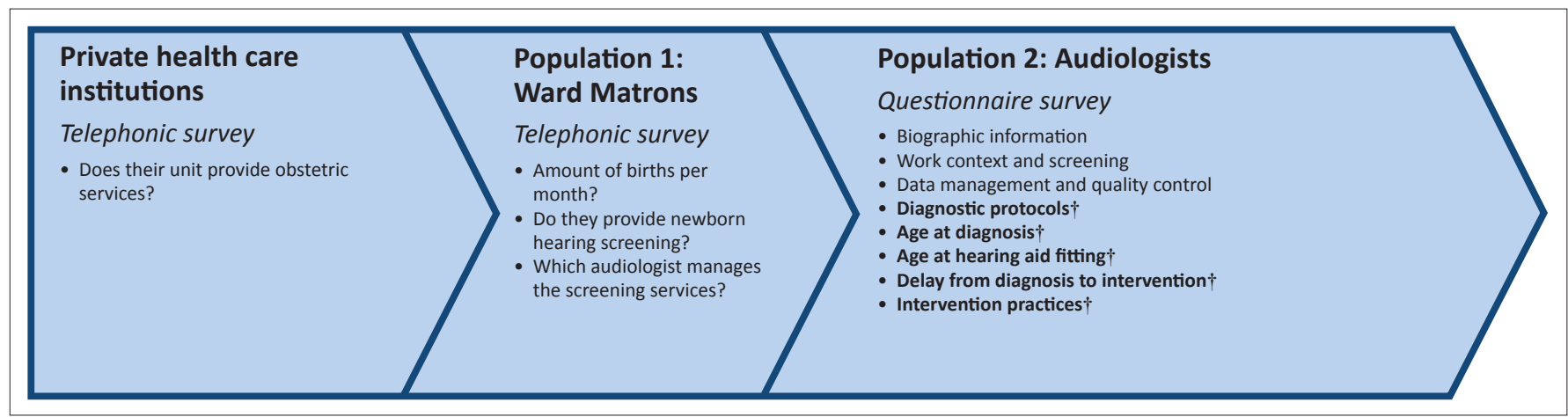

$\dagger$ Items in bold are reported in the current study.

FIGURE 1: Flowchart of study populations and survey methods.

were considered for potential inclusion in the population. After removing duplicated listings (6 listings), partially government funded institutions (8 listings) and institutions that no longer existed (4 listings), 286 institutions remained to be considered as eligible private health care sector institutions. Of these, 122 institutions did not provide obstetric services, whilst the remaining 166 did (Population 1, Figure 1). Infant hearing screening was conducted at 87 of those institutions. The 87 audiologists that offered infant hearing screening at those institutions (Population 2, Figure 1) were sent questionnaires to complete. Participants who worked at more than one private health care institution were asked to complete one questionnaire per institution to ensure that data was representative for each respective hospital or clinic.

\section{Material}

A structured telephonic survey of all private health care institutions that provided obstetric services was used for initial data collection amongst ward matrons in private health care institutions with obstetric units. Information obtained in the telephonic survey included the average amount of babies born at their institution per month, whether newborn hearing screening was conducted and who conducted it.Subsequently, a self-administered emailed or faxed questionnaire, adapted from the questionnaire used by Theunissen and Swanepoel (2008), was used for data collection. A pilot study was conducted prior to commencement of the study to improve reliability and validity. The first few sections surveyed biographical information, information on work context and hearing screening practices, data management and quality control. Survey questions relating to diagnostic protocols and intervention practices are reported on in this study.

\section{Data analysis}

Data collected from the telephonic survey with private health care institutions' obstetric ward matrons (Population 1) were linked to data collected from the questionnaires completed by audiologists (Population 2) at the respective institutions. The data was analysed to yield frequency distributions across provinces and nationally. In addition, group comparisons were made using the chi-squared and Fisher's exact tests.

\section{Results Diagnostic audiological protocols}

Of the newborn hearing screening programmes surveyed, $69 \%(53 / 77)$ provide diagnostic audiological assessment services following their screening services. Only responses by participants who provided such diagnostic audiological services were included for the diagnostic protocol results. Results indicate that in the 0-6 months category (Figure 2), most participants included distortion product oto-acoustic emissions (DPOAE) or transient emissions oto-acoustic emissions (TEOAE) (89\%; 47/53), case history and/or parental questionnaire $(85 \% ; 45 / 53)$, otoscopic examination $(66 \% ; 35 / 53)$, high-frequency tympanometry $(64 \% ; 34 / 53)$ and air conduction auditory brainstem response (ABR) (58\%; $31 / 53$ ) in their diagnostic audiological test battery.

For the 7-36 months category (Figure 2), participants generally replaced high-frequency tympanometry with standard tympanometry $(89 \% ; 47 / 53)$. More participants included behavioural observation, speech detection and speech recognition testing in testing for the older age group.

One in four participants $(26 \% ; 14 / 53)$ that provided diagnostic testing for babies $0-6$ months of age did not use auditory evoked potential testing (i.e. ABR or auditory steady state response (ASSR)). This percentage increased to $36 \%(19 / 53)$ in the older age group (7-36 months). Five participants (9\%) included ASSR for the 0-6 months age group only and not for the 7-36 months age group. A single participant included ASSR for the 7-36 months age group only and not for the 0-6 months age group. Eighty percent of participants $(61 / 76)$ were aware of the HPCSA (2007) position statement. On further analysis of the data it was found that a significant association existed between participants' years of experience and awareness of the HPCSA position statement (chi-squared and Fisher's exact tests; $p<0.05$ ). The more years of experience participating audiologists in private practice had, the less likely they were to be aware of the HPCSA position statement.

\section{Age at diagnosis}

Participants were asked to report the average number of infants diagnosed with a permanent childhood hearing loss in the previous 12 months (whether or not they conducted the 


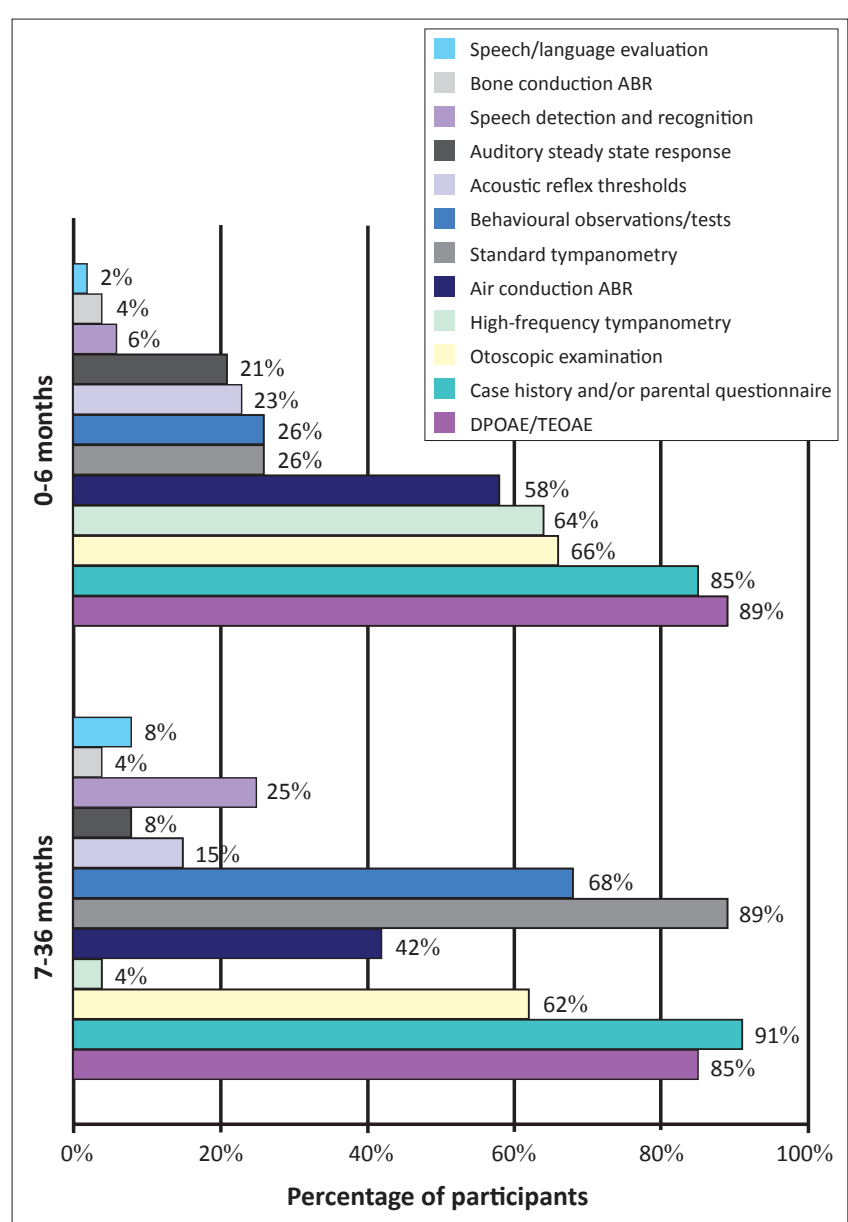

$A B R$, auditory brainstem response; DPOAE, distortion product oto-acoustic emissions; TEOAE, transient emissions oto-acoustic emissions.

FIGURE 2: Inclusion of tests in minimum diagnostic test battery for infants aged $0-6$ months and 7-36 months $(n=53)$.

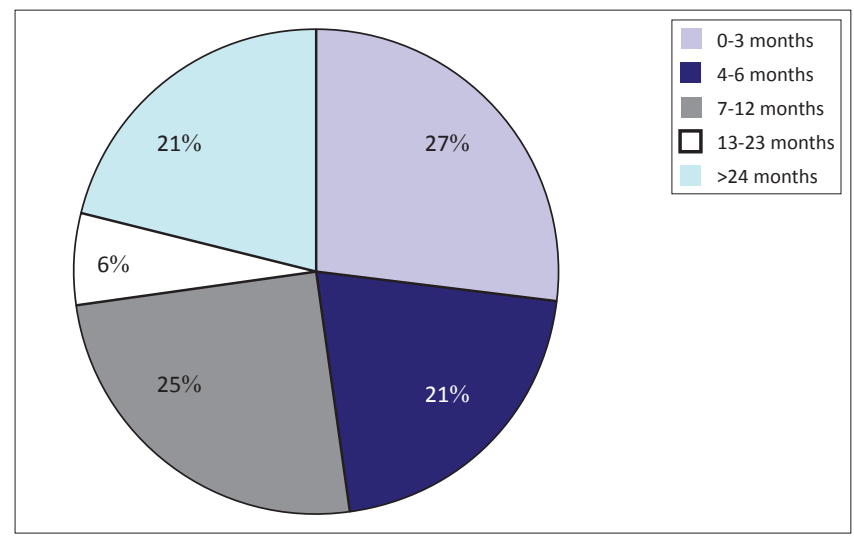

FIGURE 3: Reported average age at which diagnosis of a permanent congenital or early-onset hearing loss is confirmed $(n=48)$.

diagnostic testing themselves). The average reported numbers were added together in the two respective age group categories. Significantly more infants $(64 \% ; 145 / 227)$ were diagnosed in the $7-36$ months age group than in the $0-6$ months age group (36\%; $82 / 227)$. Most participants $(64 \% ; 35 / 55)$ indicated that they had diagnosed only 1-3 infants with a permanent childhood hearing loss in the previous 12 months.

The average reported age at diagnosis amongst the particular participants that conducted diagnostic audiological assessments ranged from 1-36 months (Figure 3). If participants did not provide diagnostic audiological testing services, their responses were not included. Twenty-seven percent $(13 / 48)$ of participants indicated that infants were diagnosed on average between 0 and 3 months of age whilst 44\% (21/48) indicated an average age at diagnosis of 9 months or older. The total average reported age at diagnosis in the private health care sector was 11 months (minimum age of 1 month; maximum age of 36 months; standard deviation of 9.98).

\section{Age at hearing aid fitting}

Most (72\%; 55/76) participants indicated that they provided hearing aid fitting services to children aged 0-36 months; however, only $84 \%$ of these $(46 / 55)$ also provided diagnostic audiological services. Only 34 of these 46 participant responses - those that were completed in full - were considered in relation to age at hearing aid fitting (Figure 4). Seventy-four percent $(25 / 34)$ indicated that less than $20 \%$ of infants who had been fitted with hearing aids at their respective private health care institutions in the previous 12 months received amplification before the age of 6 months. Fifty-nine percent of participants $(20 / 34)$ reported that less than $20 \%$ of hearing aids fitted to young children at their particular institutions in the previous 12 months was fitted between the ages of 1 and 2 years. Six participants $(18 \% ; n=34)$ indicated that an average of $81 \%-100 \%$ of children fitted with hearing aids at their respective institutions were older than 2 years.

\section{Delay from diagnosis to audiological intervention}

Most $(64 \%$; 39/61) of the participants indicated that the average period between confirmed diagnosis and hearing aid fitting (irrespective of who conducted the fitting) in their work context was 1 month. Thirteen percent (8/61), 16\% $(10 / 61), 2 \%(1 / 61)$ and $5 \%(3 / 61)$ indicated that the average waiting period is $2,3,5$ and 6 months, respectively.

The most commonly reported factors contributing to delayed audiological intervention services (Figure 5) were the family's personal funding for amplification $(62 \%$; 48/77), approval for funding from medical aids $(60 \% ; 46 / 77)$ and parents' delay in returning for follow-up appointments $(43 \% ; 33 / 77)$. The 'other' category $(10 \% ; 8 / 77)$ included factors such as transport to attend appointments, parental denial, parental resistance to accepting the diagnosis, the process of seeking a second opinion, the waiting period for donated hearing aids for orphans and the lengthy process of cochlear implant candidacy investigations.

\section{Intervention practices}

Most $(94 \%$; 72/77) of the participants indicated that they provide some form of early intervention services to children aged $0-3$ years as part of their intervention practices. Eighty percent of participants $(58 / 72)$ provide parent counselling, $77 \%$ (52/77) offer parent guidance, 50\% (36/72) provide speech-language intervention, $12 \%(9 / 72)$ offer support group services and $6 \%(5 / 72)$ provide auditory training. 


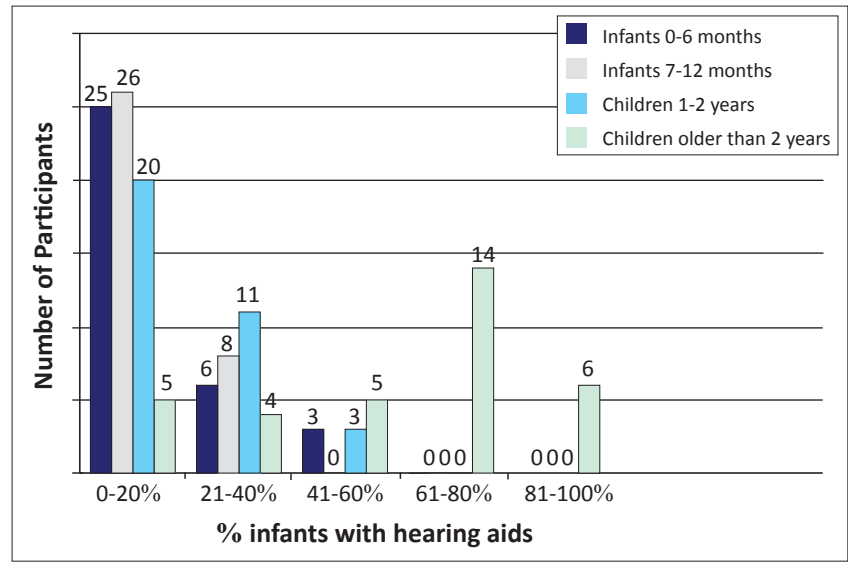

FIGURE 4: Estimated percentage of children in different age groups fitted with hearing aids in the previous 12 months $(n=34)$.

Those participants who do not provide early intervention services or provide limited services only have to refer children elsewhere for certain intervention options.

Seventy-one percent of participants (55/77) indicated that they referred children to other private practices, $70 \%(54 / 77)$ to schools or intervention centres, $45 \%(35 / 77)$ to nongovernmental organisations such as Hi Hopes (Störbeck \& Pittman, 2008) and $34 \%(26 / 77)$ to public hospitals when their services or the patient's funding are insufficient. One participant indicated having to refer children to another province as there are no available services in the vicinity, three participants reported that they refer to a cochlear implant team for intervention in possible candidacy cases, one indicated often referring to the occupational therapist and another two participants refer children and their parents to the local parent support group. None of the participants indicated that it is the parents' own responsibility to find a suitable facility that offered early intervention services.

\section{Discussion}

\section{Diagnostic audiological protocols}

Only 16\% (12/77) and 23\% (18/77) of participants included all the procedures recommended by the HPCSA (2007) position statement in their minimum diagnostic test battery for infants younger than 6 months and children 6 months and older, respectively. The most frequently occurring diagnostic procedures in participants' test batteries for infants 0-6 months included DPOAE/TEOAE, case history and/or parental questionnaire, otoscopic examination, high-frequency tympanometry and air conduction ABR. High-frequency tympanometry was replaced by standard tympanometry and air conduction ABR by behavioural observation audiometry as the most frequently occurring diagnostic procedures in test batteries for children 7-36 months of age. A similar survey in the public health care sector indicated that acoustic reflex testing was more often included in minimum diagnostic test batteries for all age groups and speech/language assessment for children 7-36 months of age (Theunissen \& Swanepoel, 2008). The public health care sector study reported that ABR testing was often omitted from minimum diagnostic test

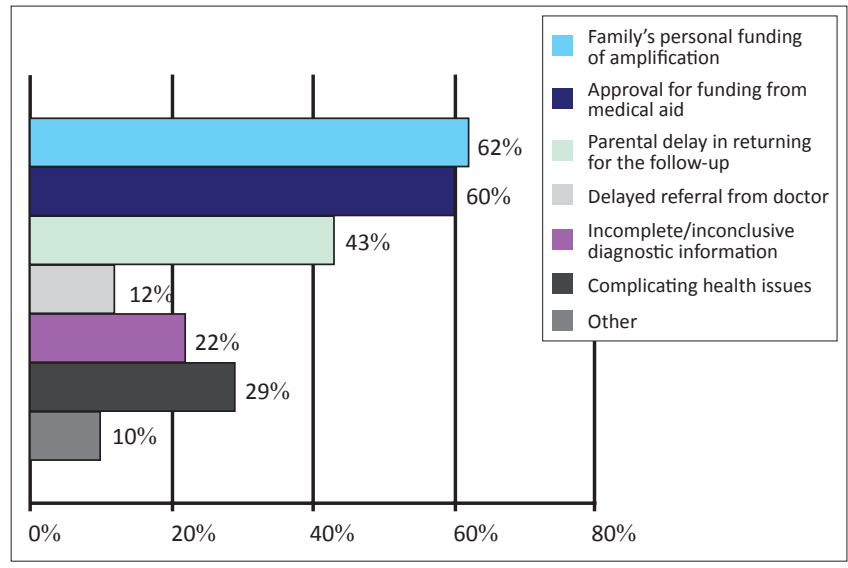

FIGURE 5: Contributing factors that prolong the waiting period between diagnosis and first visit for audiological intervention.

batteries (Theunissen \& Swanepoel, 2008). More frequent use of air conduction ABR in the private health care sector might simply be an issue of unavailability and expenses related to ABR equipment and consumables in the public health care sector (Dambisya et al., 2009; Swanepoel, 2009).

Ten participants (13\%) in the present study included all the recommended procedures (HPCSA, 2007) in their minimum diagnostic test batteries for young children (older than 6 months) except the screening of communication and language milestones. Although the screening of communication and language milestones is part of both the audiology and speech-language therapy scope of practice, it seems that audiologists in the private health care sector may rather refer to a speech-language therapist for formal assessment of speech and language. Often the speech-language therapists referred to already have experience with children with hearing impairment.

High-frequency tympanometry was omitted from the diagnostic test battery for the $0-6$ months age group by $36 \%$ $(19 / 53)$ of participants. The prevalence of otitis media with effusion in the newborn population has been reported to approximate 1 in 250 (Boudewyns et al., 2011). These infants will not be identified or receive appropriate treatment early enough without using high-frequency tympanometry (Boudewyns et al., 2011). Standard tympanometry was omitted from the diagnostic test battery for the 7-36 months age group by $11 \%(6 / 53)$ of participants. The highest prevalence rates for otitis media are between the ages of 6 and 48 months; more than $50 \%$ of children are expected to have at least one episode of otitis media with effusion in the first year of life (Boudewyns et al., 2011). This emphasises the importance of including tympanometry in the minimum test battery in order to identify otitis media with effusion promptly (Boudewyns et al., 2011).

Nine percent of participants included ASSR in their minimum test batteries for the 0-6 months age group but not for the 7-36 months age group. However, the relationship in young infants between behavioural thresholds and ASSR is not well defined and remains variable throughout the first year of 
life, especially during the first 3 weeks of life (John, Brown, Muir \& Picton, 2004; Rance, 2008). Participants might have chosen to include ASSR in the younger group as opposed to the older group as infants become increasingly active and mobile as they get older, which could make ASSR testing challenging or require conscious sedation during testing.

The variations in procedures included in diagnostic protocols amongst participants might be attributed partly to the increased equipment and consumable costs associated with certain tests, such as high-frequency tympanometry, ABR or ASSR (Swanepoel, 2009). Diagnostic tests utilised are entirely dependent on the devices acquired by audiologists, resulting in significant variability in diagnostic protocols (Meyer et al., 2012). Comprehensive test batteries in accordance with HPCSA recommended protocols are necessary not only for correct and prompt diagnosis but also for optimal amplification and verification (HPCSA, 2007; Strauss \& Van Dijk, 2008).

The variations in protocols may furthermore be ascribed in part to a lack of awareness of national and international recommendations, benchmarks and quality indicators of EHDI programmes. This study established that $80 \%$ of participants $(61 / 76)$ were aware of the HPCSA (2007) position statement, a document that specifies recommended protocols, practices and targets for EHDI programmes. However, awareness of the HPCSA position statement does not mean that recommended HPCSA protocols are implemented, as confirmed by this survey.

Non-compliance of minimum diagnostic audiological test batteries with international benchmarks and quality indicators may result in incorrect or late identification of infants with hearing impairment and subsequent suboptimal amplification (HPCSA, 2007; Korver et al., 2010; Olusanya, 2008; Olusanya \& Newton, 2007; Strauss \& Van Dijk, 2008; Yoshinaga-Itano \& Thomson, 2008). This in turn might cause delayed speech, language, cognitive, social and emotional development (HPCSA, 2007; Korver et al., 2010; Olusanya, 2008; Olusanya \& Newton, 2007; Yoshinaga-Itano \& Thomson, 2008). Ideally, paediatric diagnostic testing would be conducted at specialised paediatric facilities across the country where all necessary tests are included in the diagnostic test battery and experienced paediatric audiologists are available.

\section{Age at diagnosis}

As reported by participants, most infants and children in this private health care sector study were diagnosed with a permanent childhood hearing loss when they were older than 6 months. Less than a third $(27 \% ; 13 / 48)$ of participants indicated that infants were diagnosed with permanent childhood hearing loss at the target age (0-3 months) according to national and international benchmarks (HPCSA, 2007; JCIH, 2007; World Health Organization [WHO], 2010). The average age at diagnosis reported in this survey was 11 months. Although this does not meet the target of earliest possible diagnosis (before 3 months and no later than 4 months of age), it is better than reported in studies conducted on small urban samples in Gauteng and the Western Cape, where the average age at diagnosis was 31 and 24 months, respectively (Van der Spuy \& Pottas, 2008; Venter \& Viljoen, 2008). In a recent referral clinic study, the average age at diagnosis was 42 months (Swanepoel et al., 2013). The delayed age at diagnosis may be attributed to several factors, including poor follow-up return rates (Meyer et al., 2012; Russ, Dougherty \& Jagadish, 2010; Shulman et al., 2010; WHO, 2010), late-onset or progressive hearing loss (HPCSA, 2007; Murphy \& Radford, 2006; Shulman et al., 2010; Swanepoel et al., 2007) and poor data management systems for ensuring that the diagnostic assessment is efficient and has adequate quality control (HPCSA, 2007; Meyer et al., 2012; Shulman et al., 2010; Swanepoel et al., 2007; Swanepoel et al., 2013).

\section{Age at hearing aid fitting}

Most participants $(74 \%$; 25/34) reported that less than $20 \%$ of infants who were fitted with hearing aids in the previous 12 months received amplification by the target age of 6 months (HPCSA, 2007; JCIH, 2007). More than half (59\%) reported that most of their hearing aid fittings in the previous 12 months occurred when the children were older than 24 months. Similar suboptimal ages at hearing aid fitting were reported in previous South African studies conducted on urban samples in Gauteng and the Western Cape, where the average age at hearing aid fitting were 39 months and 28 months, respectively (Swanepoel et al., 2013; Van der Spuy \& Pottas, 2008; Venter \& Viljoen, 2008). In a national survey of the South African public health care sector, only $7 \%$ of infants who were fitted in the previous 12 months were fitted by the target age of 6 months (Theunissen \& Swanepoel, 2008). With the unsystematic hearing screening programmes and poor follow-up rates experienced in South Africa, leading to delayed diagnosis, one expects delays in hearing aid fittings as reported in the current study.

\section{Delay from diagnosis to audiological intervention}

The recommended time between confirmed diagnosis and initial hearing aid fitting is 1 month (HPCSA, 2007) and was reportedly attained by most $(64 \% ; 39 / 61)$ of the participants. The remaining participants $(36 \%)$ indicated waiting periods of 2-6 months on average. The average period between diagnosis and fitting of amplification in the national public health care sector was 5 months (Theunissen \& Swanepoel, 2008). In the public health care sector, possible factors contributing to delayed audiological intervention, including amplification, were financial, administrative or medical factors (Theunissen \& Swanepoel, 2008). It is clear that similar factors apply to the delays found in the current private health care sector study.

The most commonly reported factors contributing to delayed first visit for audiological intervention in the private health care sector were the family's personal funding for amplification or approval for funding from medical aids (financial) and 
the parents' delay in returning for follow-up appointments (administrative). The 'other' category in participant responses included factors such as transport to attend appointments (administrative) and the lengthy process of cochlear implant candidacy investigations (medical). A further category that might be added is parental acceptance of hearing impairment. Parental denial, parental resistance to accept the diagnosis and the process of seeking a second opinion were listed as significant factors that might contribute to the suboptimal waiting period for initiating intervention services. These factors are not unique to the South African context as similar factors also delay processes of EHDI programmes in the United States of America (Nelson et al., 2008; Shulman et al., 2010; Yoshinaga-Itano \& Thomson, 2008).

\section{Intervention practices}

Most (94\%) of the participants provide some form of early intervention services to children aged 0-3 years following diagnosis of permanent childhood hearing loss. Participants who do not provide early intervention services or only provide limited services indicated that they refer children elsewhere for certain intervention options. The present study did not survey the typical period between diagnosis and the commencement of early intervention services. Yoshinaga-Itano and Thomson (2008) reported that the provision of amplification is merely one of the purposes of early identification and that one should not wait to provide intervention services until the hearing aids have been acquired. The majority of participants (71\%) indicated that they refer children to other private practices for intervention (auditory training and/or speech-language therapy) - probably to speech-language therapists, as auditory training does not fall solely within the audiologist's scope of practice (Department of Health, 2012).

In one particular province in South Africa, there were no intervention services available and children had to be referred to a neighbouring province for intervention. This emphasises the need for upscaling integrated and comprehensive EHDI service infrastructure to make services accessible to all young children and their families, as well as establishing a national data management system to track young children as they progress through the system (Meyer et al., 2012; Meyer \& Swanepoel, 2011; Russ et al., 2010; Shulman et al., 2010; Swanepoel et al., 2009; Watkin \& Baldwin, 2011; WHO, 2010).

\section{Conclusion}

This study investigated the current status of diagnostic and audiological intervention services at South African private health care centres. Diagnosis of permanent congenital or early-onset hearing loss occurred earlier and delays between diagnosis and hearing aid fitting were shorter in the private health care sector than previously reported for the public health care sector (Theunissen \& Swanepoel, 2008). However, most programmes were found not to adhere to the HPCSA's (2007) diagnostic protocol recommendations, which could cause delayed or incorrect diagnoses and suboptimal amplification. The upscaling of an integrated, accessible and comprehensive EHDI infrastructure should be prioritised in South Africa in order to ensure prompt access to services and subsequent optimal benefits from the earliest possible hearing detection and intervention.

\section{Acknowledgements Competing interests}

The authors declare that they have no financial or personal relationship(s) that may have inappropriately influenced them in writing this article.

\section{Authors' contributions}

M.E.M. (University of Pretoria) acted as researcher, D.S. (University of Pretoria) as research supervisor and T.L.R. (University of Pretoria) as co-supervisor. All three authors contributed equally to writing the manuscript.

\section{References}

Blecher, M., \& Harrison, S. (2006). Healthcare financing. In P. Ijumba \& A. Padarath (Eds), South African Health Review 2006. Durban: Health Systems Trust. Retrieved June 1, 2011, from www.healthlink.org.za/uploads/files/chap3_06.pdf

Boudewyns, A., Declau, F., Van den Ende, J., Van Kerschaver, E., Dirckx, S., Hofkens Van den Brandt, A., \& Van de Heyning, P. (2011). Otitis media with effusion: An underestimated cause of hearing loss in infants. Otology \& Neurotology, 32, 799804. http://dx.doi.org/10.1097/MAO.0b013e31821b0d07

Dambisya, Y.M., Modipa, S.I., \& Health Systems Research Group, Department of Pharmacy, University of Limpopo. (2009). Capital flows in the health sector in South Africa: Implications for equity and access to health care, EQUINET Discussion Paper 76. Harare: EQUINET.

Department of Health. (2012). Government notice: Health Professions Act, 1974 (Act no. 56 of 1974): Regulations defining the scope of the profession of audiology. Government Gazette. (No. 35635).

Health Professions Council of South Africa (HPCSA), Professional Board for Speech, Language and Hearing Professions. (2007). Early hearing detection and intervention programmes in South Africa: Position Statement. Retrieved June 1, 2011, from http://www.hpcsa.co.za/Uploads/editor/UserFiles/downloads/

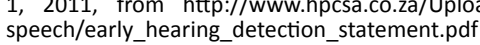

John, M.S., Brown, D.K., Muir, P.J., \& Picton, T.W. (2004). Recording steadystate responses in young infants. Ear and Hearing, 25, 539-553. http://dx.doi.org/10.1097/01.AUD.0000148050.80749.AC

Joint Committee on Infant Hearing (JClH). (2007). Year 2007 position statement: Principles and guidelines for early hearing detection and intervention programs. Pediatrics, 120(4), 899-921.

Korver, A.M.H., Konings, S., Dekker, F.W., Beers, M., Wever, C.C., Frijns, J.H.M., \&OudesluysMurphy, A.M. (2010). Newborn hearing screening vs later hearing screening and developmental outcomes in children with permanent childhood hearing impairment. Journal of the American Medical Association, 304(15), 1701-1708. impairment. Journal of the American Medical
$\mathrm{http} / / / \mathrm{dx}$.doi.org/10.1001/jama.2010.1501

Medpages. (2009). South African health professionals directory. Retrieved August 30 2009, from http://www.medpages.co.za/index.php?module=products\&category $=$ directory

Meyer, M.E., \& Swanepoel, D. (2011). Newborn hearing screening in the private health care sector - A national survey. South African Medical Journal, 101, 665-667.

Meyer, M.E., Swanepoel, D., Le Roux, T., \& Van der Linde, M. (2012). Early detection of infant hearing loss in the private health care sector of South Africa. International Journal of Pediatric Otorhinolaryngology, 76, 698-703. http://dx.doi. Journal of Pediatric Otorhino
org/10.1016/j.ijporl.2012.02.023

Morton C.C., \& Nance, W.E. (2006). Newborn hearing screening - A silent revolution The New England Journal of Medicine, 354(20), 2151-2164. http://dx.doi. org/10.1056/NEJMra050700

Murphy, E., \& Radford, L.E. (2006). Three-year outcomes of the New South Wales statewide infant screening hearing program - Australia. Newborn Hearing Screening Conference 2006, Lake Como, Italy, May 31-June 3.

Nelson, H.D., Bougatsos, C., \& Nygren, P. (2008). Universal newborn hearing screening: Systematic review to update the 2001 US Preventive Services Task Force recommendation. Pediatrics, 122, e266-e276. http://dx.doi.org/10.1542/ peds.2007-1422

Olusanya, B.O. (2008). Priorities for early hearing detection and intervention in subSaharan Africa. International Journal of Audiology, 47(Suppl 1), S3-S13. http:// dx.doi.org/10.1080/14992020802287143

Olusanya, B.O., \& Newton, V.E. (2007). Global burden of childhood hearing impairment and disease control priorities for developing countries. Lancet, 369, 1314-1317. http://dx.doi.org/10.1016/S0140-6736(07)60602-3 
Olusanya, B.O., Ruben, R.J., \& Parving, A. (2006). Reducing the burden of millennium development project. Journal of the American Medical Association, millennium development project. Journal of the American
296(4), 441-444. http://dx.doi.org/10.1001/jama.296.4.441

Rance, G. (2008). Auditory steady-state responses in neonates and infants. In G. Rance (Ed.), The auditory steady-state response (pp. 161-184). San Diego: Plural.

Russ, S.A., Dougherty, D., \& Jagadish, P. (2010). Accelerating evidence into practice for the benefit of children with early hearing loss. Pediatrics, 126, S7-S18. http:// dx.doi.org/10.1542/peds.2010-0354E

Shulman, S., Besculides, M., Saltzman, A., Ireys, H., White, K.R., \& Forsman, I. Program. Pediatrics, 126, S19-S27. http://dx.doi.org/10.1542/peds.2010-0354F

Störbeck, C., \& Pittman, P. (2008). Early intervention in South Africa: Moving beyond hearing screening. International Journal of Audiology, 47(Suppl 1), S36-S40. $\mathrm{http}: / / \mathrm{dx}$.doi.org/10.1080/14992020802294040

Strauss, S., \& Van Dijk, C. (2008). Hearing instrument fittings of pre-school children: Do we meet the prescription goals? International Journal of Audiology, 47(Suppl 1), we meet the prescription goals? International Journal
S62. http://dx.doi.org/10.1080/14992020802300904

Swanepoel, D. (2008). Infant hearing loss in developing countries - A silent health priority. Audiology Today, 20(3), 16-18.

Swanepoel, D. (2009). Early detection of infant hearing loss in South Africa. South African Medical Journal, 99(3), 158-159.

Swanepoel, D. (2010). Judith Gravel Lecture: The global epidemic of infant hearing loss - Priorities for prevention. In R.C. Seewald \& J.M. Bamford (Eds), A sound foundation through early amplification 2010: Proceedings of the Fifth Internationa Conference (pp. 19-27). Stafa, Switzerland: Phonak AG.

Swanepoel, D., Ebrahim, S., Joseph, A., \& Friedland, P.L. (2007). Newborn hearing
screening in a South African private health care hospital. International Journal screening in a South African private health care hospital. International Journal of Pediatric Otorhinolaryngology, 71, 881-887. http://dx.doi.org/10.1016/j. ijporl.2007.02.009
Swanepoel, D., Johl, L., \& Pienaar, D. (2013). Childhood hearing loss and risk profile in a South African population. International Journal of Pediatric Otorhinolaryngology 77, 394-398. http://dx.doi.org/10.1016/j.ijporl.2012.11.034

Swanepoel, D., Störbeck C., \& Friedland, P. (2009). Early hearing detection and intervention in South Africa. International Journal of Pediatric Otorhinolaryngoly, 73, 783-786. http://dx.doi.org/10.1016/j.jpporl.2009.01.007

Theunissen, M., \& Swanepoel, D. (2008). Early hearing detection and intervention services in the public health sector in South Africa. International Journal of Audiology, 47(Suppl. 1), S23-S29.

Van der Spuy, T., \& Pottas, L. (2008). Infant hearing loss in South Africa: Age of intervention and parental needs for support. International Journal of Audiology, 47(Suppl. 1), S30-S35. http://dx.doi.org/10.1080/14992020802286210

Venter, C., \& Viljoen, J. (2008). Children with hearing loss: Parental needs regarding diagnosis. Unpublished B. Communication Pathology research report, University of Pretoria, South Africa.

Watkin, P.M., \& Baldwin, M. (2011). Identifying deafness in early childhood: Requirements after the newborn hearing screen. Archives of Disease in Childhood, 96, 62-66. http://dx.doi.org/10.1136/adc.2010.185819

Watkin, P., McCann, D., Law, C., Mullee, M., Petrou, S., Stevenson, J., et al. (2007) Language ability in children with permanent childhood hearing impairment: The influence of early management and family participation. Pediatrics, 120, e694e701. http://dx.doi.org/10.1542/peds.2006-2116

World Health Organization (WHO). (2010). Newborn and infant hearing screening Current issues and guiding principles for action. Geneva: WHO Press.

Yoshinaga-Itano, C., \& Thomson, V. (2008). The work of the village: Creating a new world for children with hearing loss and their families. International Journal of Audiology, 47(Suppl. 1), S14-S22. http://dx.doi.org/10.1080/14992020802286202 